\title{
Effect of Time-Based Parameters on the Agility of a Dynamic MPC System
}

\author{
A. M. Deif, W. H. EIMaraghy (1) \\ Intelligent Manufacturing Systems Centre, Department of Industrial \& Manufacturing Systems Engineering \\ University of Windsor, Windsor, Ontario, Canada
}

\begin{abstract}
This paper presents a dynamic manufacturing planning and control (MPC) system that can maintain agility through the ability to dynamically switch between different policies due to varying market strategies. The dynamic behavior of the developed system is investigated by studying the effect of the time based parameters on responsiveness and cost effectiveness of the system reflected in the natural frequency and the damping of its different configurations. Results showed that the agility requirements are directly affected by the time based parameters of the MPC system: production lead time, capacity scalability delay, and shipment time. This resulted in a better understanding of the requirements for a well designed agile MPC system.
\end{abstract}

\section{Keywords:}

Manufacturing Planning and Control

\section{INTRODUCTION}

Agility is the measure of a manufacturer's ability to react fast to sudden and unpredictable change in demand [1]. However, this raises a question for agile MPC system about which policy is better as a buffer for demand variation, inventory or capacity? The proposed dynamic MPC model answers the question by defining agility of MPC systems as the ability to accomplish rapid and feasible dynamic changeover between the adoption of different manufacturing policies, mainly inventory based and capacity based policies, in order to adhere to the higher level management strategies dictated by market needs or trends. Studying the behavior of these systems is better achieved via dynamic modeling and analysis approaches since am agile environment is a dynamic one with a high degree of uncertainty.

The focus of this paper is to study the effect of timebased parameters of the developed MPC system (mainly the production lead time, capacity scalability delay time and shipment time) on the performance of the developed MPC system. This is achieved from a dynamic perspective by investigating the effect of these parameters on the natural frequency and the damping ratio of the transfer functions of the different configurations (policies) of the developed MPC system.

\section{LITERATURE REVIEW}

Previous research that considered dynamic analysis of MPC systems included the application of system dynamics approaches to control capacity as in Hello [2] and inventory and WIP as in Fowler [3]. Hillon and Porth [4] used discrete event simulation to control production in job shop systems. In addition, the application of nonlinear dynamic analysis to understand the dynamic behavior of MPC systems was considered by Radons and Neugebauer [5] to control the flow of products in a production system and by Scholz-Reiter and Schmieder [6] to control different production parameters. Chaos theory, as a dynamic analysis approach, was also applied by Chryssolouris et al. [7] to solve scheduling problems.
For more insight into the analysis, design and control of MPC systems, control theoretic approaches have been proposed. Using these approaches, Wiendahl [8] developed the funnel model of manufacturing systems and was used to control WIP and backlog [9]. A model developed by Duffie and Falu [10] for a single workstation closed loop PPC was used to control WIP and backlog in [11] and [12]. The model was extended to a multiple work-stations by Kim and Duffie [13]. Asl and Ulsoy [14] applied closed loop techniques to control capacity scalability in reconfigurable manufacturing systems. Control approaches were also used in inventory control applications, especially using the APIOBPCS (Automatic Pipeline, Inventory and Order Based Production Control System) model developed by John et al. [15]. Examples include application to supply chain management [16] and adaptive inventory control [17].

The main MPC dynamic variables considered in the past work were capacity, WIP and inventory. However, none of these dynamic models linked the three variables together. In an agile environment, capacity and inventory can be used to hedge for demand variation based on the market trend. The proposed MPC system achieves its agility by linking these parameters together in one model controlled by a central decision logic unit.

\section{THE DYNAMIC AGILE MPC MODEL}

The dynamic modeling of the agile MPC system aims at constructing a model, in which different planning and control configurations can be realized with respect to a higher level strategy. The system model shown in figure 1 includes the three main controlled variables that can work individually or two at a time based on the decision of the supervisory controller (the decision logic unit) to determine the desired production rate (DPR). The variables are the work in process (WIP), the capacity rate of the system and the finished inventory level.

The proposed general structure of the agile MPC system can be described as being composed of two main operational layers in addition to a decision logic unit that links these two layers with the higher management layer. 


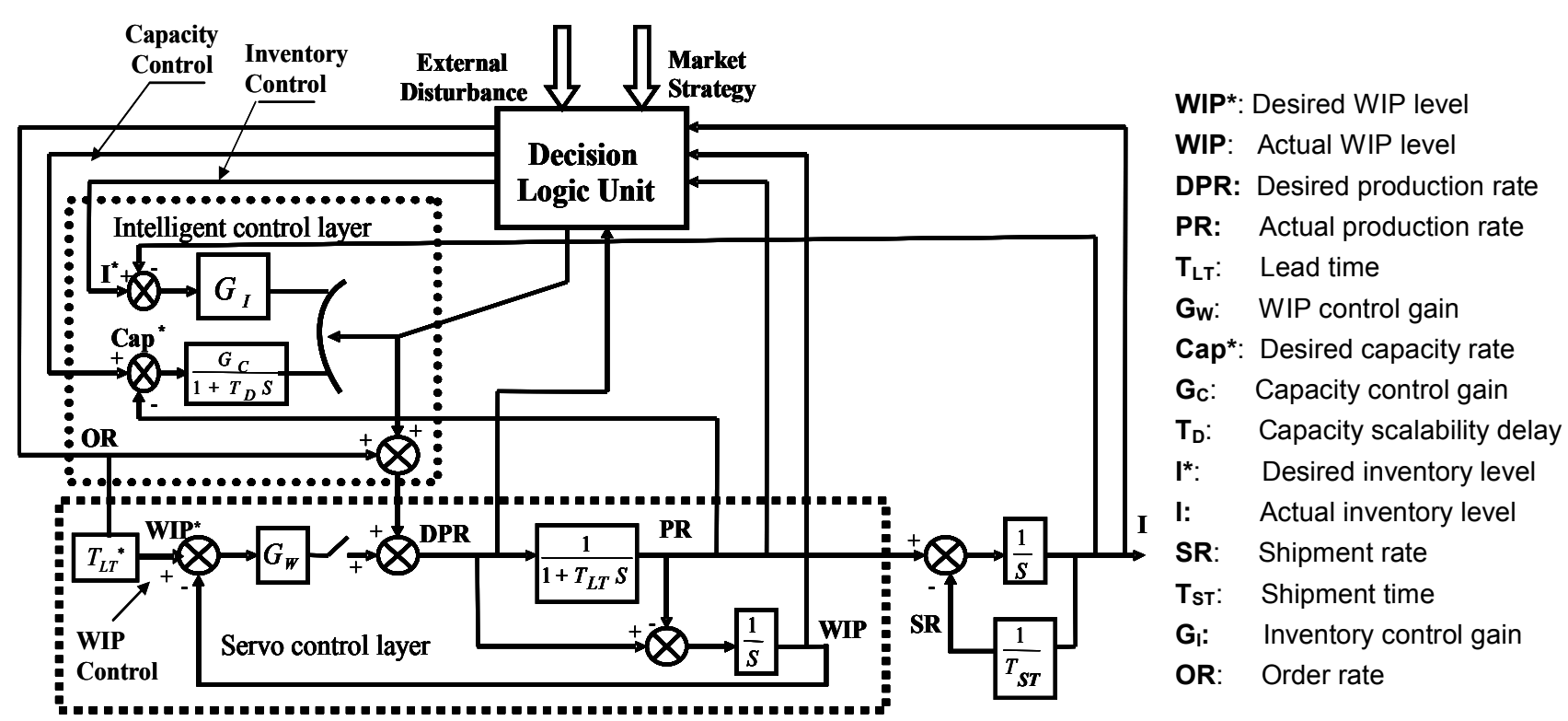

Figure 1: Agile MPC system model.

The first operation layer is the default (or servo control) layer where the control of the manufacturing system is only based on controlling the WIP level. This it is similar to the WIP model developed John et al. [15]. The other layer (intelligent control layer) involves two controllers, an inventory controller and a capacity rate controller. Either can work with the servo control layer or by itself, creating different MPC configuration or policies. This is decided by the decision logic unit (supervisory controller). Furthermore, the decision logic unit provides the system with the reference control points and the updates of the order rate (OR) and shipment time ( $\left.T_{S T}\right)$ based on demand data from the higher management level and at the same time collects all the data of the current system to help in deciding for the optimal MPC configuration. The production process is modeled as a pipeline where outflow is lagged by lead time, $T_{L T}$.

Equations (1) to (4) list the transfer functions for the developed agile MPC system configurations. Without losing the generality, two basic assumptions were made. First, the expected lead time is assumed to be equal to the actual one $\left(T_{L T}=T_{L T}{ }^{*}\right)$. Second, the shipment rate is set to be equal to the order rate $(S R=O R)$. These assumptions are made only for better understanding the problem. The proposed model does not have any limitations with respect to considering the case of any linear or non-linear relation between the variables.

Capacity Based MPC System:

$$
\frac{P R}{\operatorname{Cap}^{*}}=\frac{G_{C} T_{L T}{ }^{-1} T_{D}^{-1}}{S^{2}+S\left(T_{L T}{ }^{-1}+T_{D}{ }^{-1}\right)+\left(1+G_{C}\right) T_{L T}{ }^{-1} T_{D}{ }^{-1}}
$$

Finished Inventory Based MPC System:

$$
\frac{I}{I^{*}}=\frac{G_{I} T_{L T}{ }^{-1}}{S^{2}+S\left(T_{L T}{ }^{-1}+T_{S T}{ }^{-1}\right)+G_{I} T_{L T}{ }^{-1}}
$$

Capacity/WIP Based MPC System:

$$
\frac{P R}{C a p^{*}}=\frac{G_{W}\left(T_{D}^{-1}+S\right)+G_{C} T_{L T} T^{-1} T_{D}^{-1}}{S^{2}+S\left(T_{D}^{-1}+T_{L T}^{-1}+G_{W}\right)+\left(G_{W} T_{L T}+G_{C}+1\right) T_{L T}{ }^{-1} T_{D}^{-1}}
$$

Finished Inventory/WIP Based MPC System:

$$
\frac{I}{I^{*}}=\frac{G_{I} T_{L T}{ }^{-1}}{S^{2}+S\left(G_{W}+T_{L T}{ }^{-1}+T_{S T}{ }^{-1}\right)+\left(G_{I} T_{L T}{ }^{-1}\right)}
$$

\section{DYNAMIC ANALYSIS OF MPC MODEL USING NATURAL FREQUENCY AND DAMPING RATIO}

Responsiveness and cost effectiveness are the major objectives of any agile MPC system. From a dynamic stand point, these two objectives can be expressed through some dynamical characteristics of the developed model. Responsiveness of the agile MPC system can be investigated by exploring the rise time of the system when subjected to sudden change in demand. The smaller the rise time is, the faster the system response. The settling time can also give an idea about the time required for the system to reach stable production which is also tied to responsiveness. As for cost effectiveness, a dynamic characteristic that can express an aspect of that objective is the overshooting of the system when subjected to sudden demand change as it describes the amount of extra production (or inventory) that the system encounters to respond to that change.

The previous dynamic characteristics, as all other transient time characteristics of a second order transfer function, can be expressed as a function of the natural frequency and the damping ratio of the system. Therefore, the term "MPC system's natural frequency" can be used to measure the responsiveness of the system to external excitation (demand). The higher the natural frequency, the more responsive the system is. This can be further explained by examining the units of the MPC system's natural frequency which are production cycles/production time (time can be hours, days or shift). Thus the higher the MPC system's natural frequency the smaller the time a production cycle needs to be completed. The damping ratio of the MPC system reflects the different system's parameters that damp the production's (or inventory's) oscillation and can act as absorbers to sudden changes in demand or various internal disturbances. Therefore, it gives an insight about the relative stability of the MPC system. In this paper some analysis of the agility of the developed MPC system will be conducted through exploring the effect of the time based parameters of the system over these two quantities. Table 1 lists the natural frequency and the damping ratio of the different MPC system's policies as a function of the different MPC system's parameters.

The considered time based parameters are: production lead time, the capacity scalability delay time which is the time required to scale capacity rate together with the ramp up time for the new scaled system; and finally the shipment time which is used to express the shipment rate and is indicated based on the market strategy. 


\begin{tabular}{|c|c|c|}
\hline $\begin{array}{l}\text { MPC } \\
\text { System } \\
\text { Policy }\end{array}$ & $\begin{array}{c}\text { Natural } \\
\text { Frequency }\end{array}$ & Damping Ratio \\
\hline Inventory & $\omega_{\mathrm{n}}=\sqrt{\frac{G_{I}}{T_{L T}}}$ & $\xi=\frac{1}{2 \omega_{n}}\left(\frac{1}{T_{L T}}+\frac{1}{T_{S R}}\right)$ \\
\hline Capacity & $\omega_{\mathrm{n}}=\sqrt{\frac{1+G_{C}}{T_{L T} T_{D}}}$ & $\xi=\frac{1}{2 \omega_{n}}\left(\frac{1}{T_{L T}}+\frac{1}{T_{D}}\right)$ \\
\hline $\begin{array}{c}\text { Inventory/ } \\
\text { WIP }\end{array}$ & $\omega_{\mathrm{n}}=\sqrt{\frac{G_{I}}{T_{L T}}}$ & $\xi=\frac{1}{2 \omega_{n}}\left(\frac{1}{T_{L T}}+\frac{1}{T_{S R}}+G_{W}\right.$ \\
\hline $\begin{array}{l}\text { Capacity/ } \\
\text { WIP }\end{array}$ & $\omega_{\mathrm{n}}=\sqrt{\frac{G_{W} T_{L T}+G_{C}+1}{T_{L T} T_{D}}}$ & $\xi=\frac{1}{2 \omega_{n}}\left(\frac{1}{T_{L T}}+\frac{1}{T_{D}}+G_{W}\right.$ \\
\hline
\end{tabular}

Table 1: Natural frequency and damping ratio of the different agile MPC system's policies.

\section{AGILE MPC SYSTEM APPLIED TO AUTOMATIC PCB ASSEMBLY LINE}

The developed agile MPC system was applied to a small automatic PCB assembly line dedicated for the assembly of certain computer peripherals. The market demand was usually in the order of $36^{*} 10^{3}$ of peripherals. However, as most of the electronic products, the market demand is always subjected to fluctuations. The line is composed of 2 pick and place machines with average throughput of $6 * 10^{3} /$ day for each machine ( $T_{L T}=3$ days for an order). Only one extra machine with throughput of $4^{*} 10^{3}$ can be added to the line $\left(\mathrm{G}_{\mathrm{C}}=4^{*} 10^{3} /\right.$ day $)$ and needs 2 days for installation and alignment $\left(T_{D}=2\right.$ days). The line is designed to have a maximum WIP of $1 * 10^{3}$ $\left(G_{W}=1 * 10^{3} /\right.$ day). The maximum input rate increase within the normal line capacity is $3 \mathrm{~K} /$ day of bare $\mathrm{PCB}$ and SMD components kits $\left(\mathrm{G}_{1}=3^{\star} 10^{3} /\right.$ day). The shipment of the finished orders is carried out every 3 days ( $T_{S R}=3$ days).

Figures 2 and 3 show the effect of production lead time on natural frequencies and damping ratios for the different MPC configurations (policies). It is clear that as the production lead time increases, the MPC natural frequency of the different configurations decreases indicating a lower level of responsiveness. This is due to longer production times.

As for the damping ratio, it increases as the lead time increases, indicating a higher level of stability since the system has enough time to respond to sudden demand changes and therefore production cost is reduced. It is also important to note that this increase in the damping ratio is more significant in MPC polices that compensate for the WIP level due to the damping effect of the WIP in any production line.

In the capacity based MPC configuration, the damping ratio of the system decreases as the lead time increases when the lead time values are less than the capacity scalability delay time. The same observation applies to inventory based MPC system when manufacturing lead times are less than the shipment time. In both policies the damping ratio maintains its minimum when the manufacturing lead time is equal to the capacity scalability delay time (in capacity based MPC) or is equal to shipment time (in inventory based MPC policy) as it is the case when adopting just in time (JIT) policies. This observation is important when designing the MPC system to have a certain lead time while considering the stability level (reflected by damping ratio) and cost of production (reflected by production overshooting).

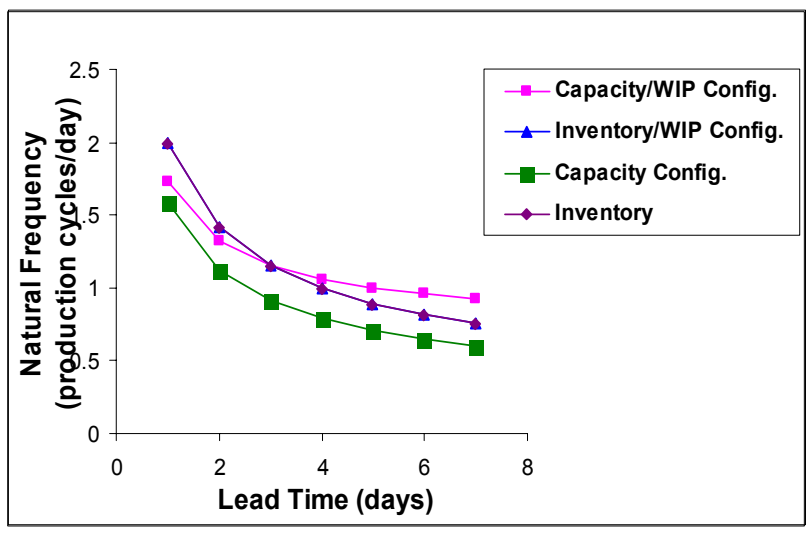

Figure 2: Effect of lead time on the natural frequency of MPC configurations.

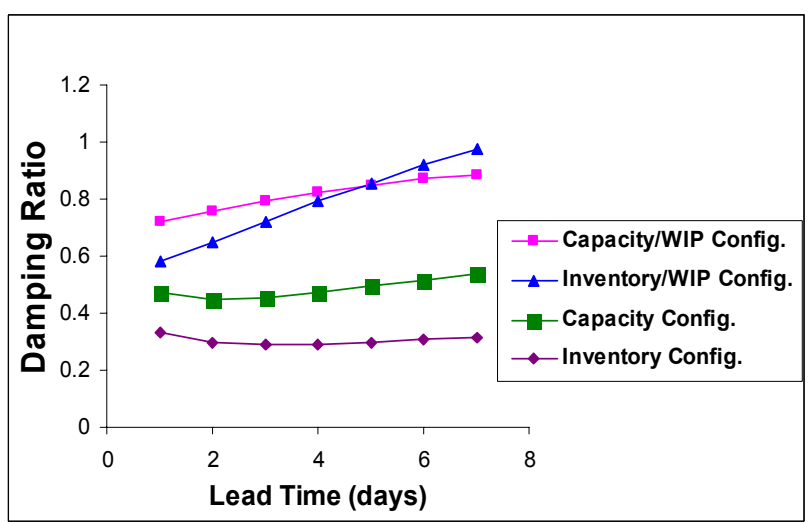

Figure 3: Effect of lead time on the damping ratio of MPC configurations.

Figures 4 and 5 show the effect of capacity scalability delay time in capacity based MPC configurations. The same relation between the production lead time and the natural frequency of the MPC system is observed with the capacity scalability delay time. This can be expected since scaling the capacity plus the ramp up time of the system after being reconfigured in general add to the overall production cycle time leading to the decrease of the responsiveness level of the system.

The damping ratio of the MPC policy also behaves similarly with the capacity scalability delay time as with the production lead time. The damping effect of the WIP is obvious when comparing the increase of the damping ratio between the two capacity based policies.

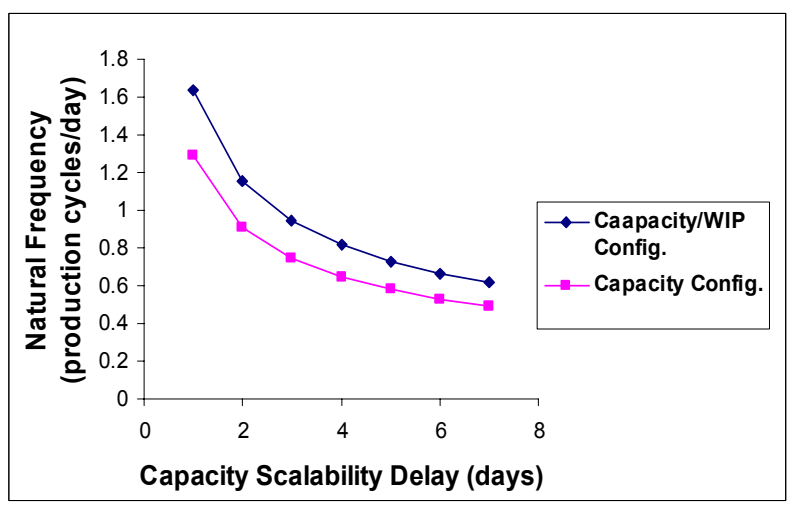

Figure 4: Effect of capacity scalability delay time on the natural frequency of capacity based MPC configurations. 


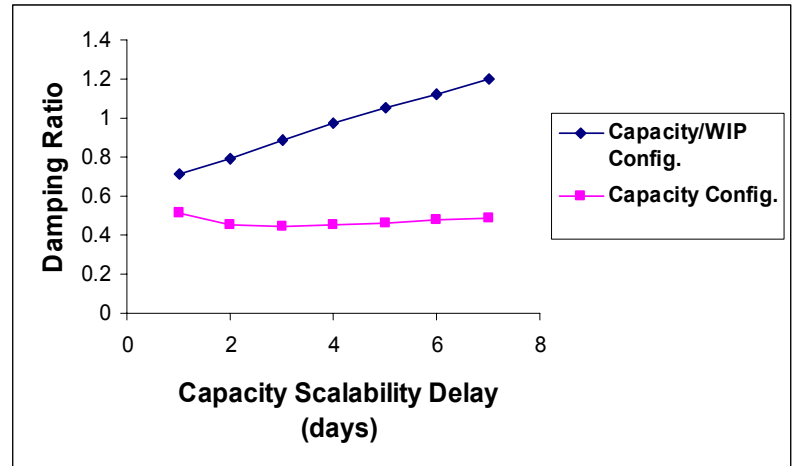

Figure 5: Effect of capacity scalability delay time on the damping ratio of capacity based MPC configurations.

In inventory based MPC policies, the shipment time does not affect the MPC's natural frequency. From a manufacturing perspective, this can be explained since these policies are usually used when the enterprise is adopting a push market strategy and higher management level decides the amount to be pushed to market and in turn the required production rate, which is reflected in the system's natural frequency.

Figure 6 shows the effect of the shipment time on the damping ratio for inventory based MPC systems. It is clear that as shipment time increases, the damping ratio of the system decreases, indicating less effort to respond to market changes. This can be explained by the fact that the longer the shipment time, the lower the rate of products to be pushed to market. This means that the system does not need a high damping effort to respond to market changes since there is enough span of time for that. Also, it is shown that the decrease in the damping ratio is much higher when the shipment time is less than the lead time ( 3 days in this case). This observation helps in designing MPC system parameters based on the required damping level.

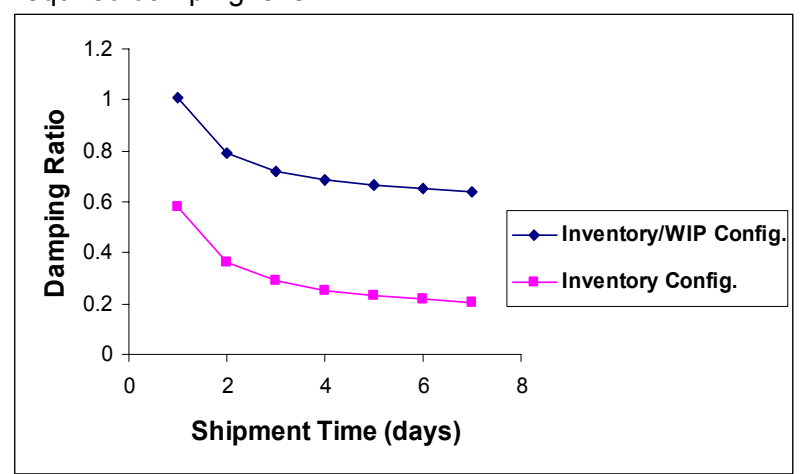

Figure 6: Effect of shipment time on the damping ratio of inventory based MPC configurations.

\section{CONCLUSIONS}

An agile MPC system was proposed, and analyzed using control theoretic approaches. The analysis aimed at exploring the effect of the time based parameters of the system on the main agility objectives, responsiveness and cost effectiveness. These objectives were shown respectively to be reflected in the natural frequency and the damping ratio of the different configurations of the developed MPC system.

Results showed that production lead time and capacity scalability delay time are inversely proportional to the natural frequency of the MPC system and lead to the decrease of the responsiveness level. This confirms the known fact that to have a successful implementation of capacity based MPC systems (like in the case of RMS) effort should be made to decrease this delay. The damping ratio of capacity based MPC policies were directly proportional to the lead time and scalability delay time, indicating that both times enhance the robustness of the system, which positively affect the production cost. In inventory based policies, the shipment time had no effect on the natural frequency of the system while it decreased its damping ratio. The damping effect of the WIP was clear while examining the damping ratio of MPC polices that considered WIP compensation.

Further work is needed to examine the effect of other parameters of the agile MPC system, especially the controllers' gains values. Finally, the design of the decision logic unit that optimally masters the different MPC system's configuration is important for the practical implementation of the proposed model.

\section{REFERENCES}

[1] Noaker, P., 1994, Search for Agile Manufacturing, Manufacturing Engineering, Nov: 40-43.

[2] Helo, P., 2000, Dynamic Modeling of Surge Effect and Capacity Limitation in Supply Chains, Intl. Journal of Production Research, 38/17:4521-4533.

[3] Fowler, A., 1999, Feedback and Feedforward as Systematic Framework of Operation Control, Intl. Journal of Operation Management, 19/2:182-204.

[4] Hillon, H., Proth, J., 1989, Performance Evaluation of Job-Shop Systems Using Event-Graph, IEEE Transactions on Automatic Control, 34/1:3-9.

[5] Radons, G., Neugebauer, R., 2005, Nonlinear Dynamics of Production Systems, Willy- $\mathrm{VCH}$.

[6] Scholz-Reiter, B., Schmieder, F., 2002, Modeling and Control of Production Systems Based on Nonlinear Dynamics Theory, Annals of the CIRP, 51/1:375-379.

[7] Chryssolouris, G., Giannelos, N., Papakostas, N., Mourtzis, D., 2004 Chaos Theory in Production Scheduling, Annals of the CIRP, 53/1:3-6

[8] Wiendahl, H.P., 1995, Load Oriented Manufacturing Control, Springer-Verlag: Hanover, $\mathrm{NH}$.

[9] Wiendahl, H.P., Breithaupt, J., 2001, BacklogOriented Automatic Production control, Annals of the CIRP, 43/2:533-540.

[10] Duffie, N., Falu, I., 2002, Control-theoretic Analysis of a Closed Loop PPC System. Annals of the CIRP, 52/1:379-382.

[11] Ratering, A., Duffie, N., 2003, Design and Analysis of Closed-Loop Single-Workstation PPC System, Annals of the CIRP, 52/1:355-358.

[12] Kim, J-H., Duffie, N., 2004, Backlog Control Design for a Closed Loop PPC System, Annals of the CIRP, 53/1: 357-360

[13] Kim, J-H., Duffie, N., 2005, Design and Analysis for Closed Loop Capacity Control of a Multi Workstation Production System, Annals of the CIRP, 54/1:470-474.

[14] Asl, F., Ulsoy, A., 2002, Capacity Management via Feedback Control in RMS, Proceeding of Japan/USA Symposium on Flexible Manufacturing Automation, Hiroshima, Japan.

[15] John, S., Towill, D., Naim, M., 1994, Dynamic Analysis of a WIP Compensated Support System, Intl. Journal of Manufacturing System Design, 1: 283-297.

[16] Diseny, S., Towill, D., 2002, A Discrete Transfer Function Model to Determine Dynamic Stability of VMI in Supply Chain, Intl. Production Research, 40/1:179-204.

[17] Towill, D., Evans, G., Chema, P., 1997, Analysis of an Adaptive Reasonable Inventory Control System, Journal of Production Planning and Control, 8/6:545-557. 